
\title{
28 Research Square \\ Gut Microbiota Composition and Functional Prediction in Recurrent Spontaneous Abortion
}

\section{Ying Cui}

Jiangxi Maternal and Child Health Hospital

Li Zou

Jiangxi University of Traditional Chinese Medicine

Qian Ye

Jiangxi University of Traditional Chinese Medicine

Dandan Li

Jiangxi University of Traditional Chinese Medicine

Huiming Wu

Jiangxi University of Traditional Chinese Medicine

Ling He ( $\square$ heling118@126.com )

Jiangxi University of Traditional Chinese Medicine

\section{Research Article}

Keywords: Gut microbiota composition , functional prediction, recurrent spontaneous abortion, microbial community, pregnant women

Posted Date: October 22nd, 2021

DOl: https://doi.org/10.21203/rs.3.rs-906730/v1

License: (c) (i) This work is licensed under a Creative Commons Attribution 4.0 International License.

Read Full License 


\section{Abstract}

\section{Objective}

The changes of microbial community in pregnant women, let alone those of patients with recurrent spontaneous abortion (RSA), remain unclear. We analyzed the differences of gut mircobiota (GM) between RSA patients and pregnant women to find the possible mechanism of RSA.

\section{Methods}

We enrolled 30 RSA patients (RSA group) and 30 pregnant women who terminated their pregnancy and did not have a history of spontaneous abortion (NR group) in our hospital from June 2020 to August 2020, and fecal samples were obtained to analyze the GM using 16S rDNA V3-V4 sequencing.

\section{Results}

At the phylum level, we found that there is no significant difference in composition of GM between RSA and NR. But at the genus level, compared with NR, Roseburia significantly decreased $(P<0.01)$, and Ruminococcus significantly increased in RSA patients $(P<0.05)$. Further analysis indicated that Klebsiella $(P<0.05)$ was significantly increased, Prevotella.9 $(P<0.05)$ and Roseburia $(P<0.05)$ were significantly decreased in RSA2 group (BMl>23.9 in RSA). Moreover, Agathobacter $(P<0.01)$ was significantly increased in NR2 group (no delivery in NR). Functional prediction indicated that GM may interfere with RSA through membrane transport, carbohydrate metabolism, amino acid metabolism and other pathways.

\section{Conclusion}

Decreased Roseburia in GM of pregnant women maybe related to RSA. Our results provide the basis for in-depth studies of the composition of gut microbial communities in RSA.

\section{Background}

The microbial communities of humans are characteristic and complex mixtures of microorganisms that have co-evolved with their human hosts [1]. Microbes colonize the neonatal gut immediately following birth [2], maternal factors are important determinants of early infant colonization, many studies indicated that maternal microbiota have an important impact on infant gut microbiota, as prevention of allergic diseases, affect the infant's immunity, and so on $[3,4,5]$. The composition and development of infant gut microbiota can be influenced by many prenatal factors, such as maternal diet, obesity, smoking status, and use of antibiotic agents during pregnancy [6]. In addition, the mode of delivery, type of infant feeding, gestational age, infant hospitalization were considered to be important determinants of the gut microbiotic composition in infants [7]. 
In view of the important influence of microbiota on the mother and fetus, some scholars have studied the microbiota in natural cavities of pregnant women, such as the oral cavity, vagina, uterus and gut, to observe their influence on the pregnancy outcome. Recently, based on 16SrDNA sequencing, several studies have shown that the structure and stability of maternal microbiota changed during pregnancy, for example, Lactobacillus in the maternal vagina and intestinal tract have a potentially important impact on fetal development and may affect the outcome of pregnancy. But the results of these studies varied widely, which possibly due to the differences in race, diet, antibiotic use and education level [8-12]. The research on the gut microbiota (GM) of pregnant women focuses on the aspects of pregnant women with diabetes mellitus, pre-eclampsia and [13-14].

Recurrent spontaneous abortion (RSA) is a common gynecological disease in China, defined by two or more failed pregnancies, its etiology remains unclear, genetics, autoimmune abnormalities, endocrine, anatomy, or pre-thrombotic state are thought to be related to its pathogenesis $[15,16]$. Previous proteomic study had shown that there is a significant changes occurred in the pathway of Fc gamma R-mediated phagocytosis in RSA, this result indicated that autoimmunity is invloved in its pathogenesis [17]. A study suggested that RSA may be caused by an imbalance of vaginal flora (especially that of Pseudomonas) in RSA patients [18]. In this study, we focused on the relationship of maternal GM and RSA. So far, no such study has been reported.

Through this study, we want to explain the following questions: 1) Whether there is a difference in GM between RSA patients and normal abortion patients, whether there is statistical significance; 2) Is the maternal gut microbiota related to RSA, and if so, which bacteria play an important role? 3) How these bacteria invloved in mechanisms of RSA and whether it is related to body mass index (BMI).

\section{Methods}

\section{Study design}

From June 2020 to August 2020, in the case group (RSA), we recruited 30 RSA patients who met the diagnostic criteria of Royal College of Obstetricians and Gynecologists (RCOG) consensus [16].

Meanwhile, in the control group (NR), we recruited 30 women who terminated their pregnancy and did not have a history of spontaneous abortion. Exclusion criteria for all subjects included: 1) Complicate with chromosomal abnormalities, anatomical abnormalities, surgery and trauma, etc; 2) taking antibiotics, probiotics, or other treatments, within 4 weeks; 3 ) Complicate with endocrine diseases, infectious diseases, immune diseases; 4) any psychiatric comorbidity; 5) excessive physical exercise. Characteristics of participants were summarized in Table 1.

\section{Sample collection and DNA Extraction}

A single fecal sample was collected by each participant at home, and immediately stored $a t-20^{\circ} \mathrm{C}$, then transferred to $-80^{\circ} \mathrm{C}$ for longer-term storage. Fecal bacteria genomic DNA was extracted with 


\section{S rDNA gene sequencing}

The following steps and methods refer to the implementation method of Lundberg DS [19].

\section{Amplicon Generation:}

Primer: 16S V3-V4: 341F-806R. 16S rDNA genes were amplified used the specific primer with the barcode. All PCR reactions were carried out in $30 \mu \mathrm{L}$ reactions with $15 \mu \mathrm{L}$ of Phusion ${ }^{\circledR}$ High-Fidelity PCR Master Mix (New England Biolabs).

\section{PCR Products treatment:}

PCR products were mixed with same volume of $1 X$ loading buffer (contained SYB green), electrophoresis were operated on $2 \%$ agarose gel fordetection, mixture PCR products were purified with AxyPrepDNA Gel Extraction Kit (AXYGEN).

\section{Library preparation and sequencing:}

Following manufacturer's recommendations, sequencing libraries were generated using NEB Next ${ }^{B}$ Ultra ${ }^{T M}$ DNA Library Prep Kit for Illumina (NEB, USA) and index codes were added. The quality of library was assessed on the Qubit@ 2.0 Fluorometer (Thermo Scientific) and Bioanalyzer 2100 system (Agilent). Then, paired-End (PE) amplicon library was constructed using a TruSeq ${ }^{\circledR}$ DNA PCR-Free Sample Preparaion Kit (IIlumina, US) and quantified by Qubit, then sequencing was performed using the Illumina Hiseq platform (APTBIO Technology, Shanghai, China).

\section{Data and bioinformatics analysis}

The following steps and methods refer to the implementation methods of Lundberg DS [19] and Avershina $E[20]$.

\section{Paired-end reads assemblies:}

Paired-end reads from the original DNA fragments were merged using FLASH (http://ccb.jhu.edu/software/FLASH/), Quality fltering on the raw tags was performed under specifc flering conditions to obtain high-quality. clean tags according to the Fast QC (V 0.10.1). 


\section{OTU cluster and Species annotation:}

Chimeric sequences were fltered using Useach sofware (10 version). Sequences with $97 \%$ similarity were assigned to the same operational taxonomic units (OTUs) by Usearch (10 version). Representative sequences were chosen for each OTU, and taxonomic data were then assigned to each representative sequence using the RDP (Ribosomal Database Project) classifer. Sequences were processed with the sofware package of the QIIME (V1.9.0) toolkit. Taxonomy-based analyses were conducted by classifying each sequence using the SILVA database (https://www.arb-silva.de/). In order to compute Alpha Diversity, we rarify the OTU table and calculate seven indexes: Ace, Chao1, Goods coverage, Observed Species, PD whole tree, Shannon, Simpson.

\section{Phylogenics distance and community distribution:}

Graphical representation of the relative abundance of bacterial diversity from phylum to species can be visualized using Krona chart. Cluster analysis was preceded by principal component analysis (PCA), which was applied to reduce the dimension of the original variables using the QIIME (Version 1.9.1) software package. QIIME calculates both weighted and unweighted unifrac distance, which are phylogenetic measures of beta diversity. We used unweighted unifrac distance for Principal Coordinate Analysis (PCoA) and Unweighted Pair Group Method with Arithmetic mean (UPGMA) Clustering.

\section{Statistical analysis:}

STAMP software was utilized to confirm differences in the abundances of individual taxonomy between the two groups. LDA Effect Size (LEfSe) was used for the quantitative analysis of biomarkers within two groups. To identify differences of microbial communities between the two groups, ANOSIM and ADONIS were performed based on the Bray-Curtis dissimilarity distance matrices.

\section{Results}

\section{The number of OTUs}

The number of common OTUs between the patients of RSA and NR was 23672. Meanwhile, the number of proper OTUs in the patients of RSA was 17847, and that in NR was 43885, as shown in Table 1.

\section{Table 1}

\section{Characteristics and OTUs of two groups}




\begin{tabular}{|llll|}
\hline Group & RSA & NR & P-value \\
\hline Number & 30 & 30 & $/$ \\
\hline Age & $30.00 \pm 1.41$ & $28.03 \pm 3.54$ & 0.11 \\
\hline Body mass index (BMI) & $21.74 \pm 0.35$ & $20.58 \pm 2.09$ & 0.07 \\
\hline The number of spontaneous abortions & & \\
\hline 2 times & 18 & $/$ & $/$ \\
\hline more than 3 times & 12 & $/$ & $/$ \\
\hline OTUs & 41529 & 77577 & $/$ \\
\hline
\end{tabular}

\section{Characterization of fecal microbiota}

The phylum and genus level were choosed to show a histogram of relative abundance of species. As shown in Figure 1, at the phylum level, we found that there is no significant difference in composition of GM between RSA and NR. But at the genus level, the results were shown in Figure 2 that compared with NR, Roseburia significantly decreased $(P<0.01)$, and Ruminococcus significantly increased in RSA patients $(P<0.05)$.

The normal group was divided into two groups according to whether to give birth or not, those who did not give birth were included in NR2. According to BMI, uterine and ovarian lesions, patients with intrauterine adhesion, polycystic ovary syndrome (PCOS), and BMI > 23.9 among RSA patients were classified as RSA1 group, and the remaining patients were classified as RSA2 group. We analyzed the relative abundance of different groups again. As shown in Figure 3 , the results indicated that at the genus level, Klebsiella $(P<0.05)$ were significantly increased, Prevotella. $9(P<0.05)$ and Roseburia $(P<0.05)$ were significantly decreased in RSA1 group. Moreover, Agathobacter was significantly increased in NR2 group $(P<0.01)$.

NR1 was pregnant women who had given birth, NR2 was pregnant women who had not given birth, RSA1 was RSA patients with intrauterine adhesion, PCOS, and BMI > 23.9, RSA2 was other RSA patients.

\section{Alpha diversity}

Alpha diversity is used to analyze the microbial community diversity within samples or groups. Observed species is the number of OUT, Shannon and Simpson were the bacterial diversity index, Chao1 and ACE are the bacterial abundance index. Coverage was the depth index of sequencing. PD whole tree is the phylogenetic diversity index. Comparison of alpha diversity difference indexes between RSA and NR was 
shown in Table 2. The results of difference analysis of alpha diversity between two groups were represented by a boxplot (Figure 4).

\section{Table 2}

\section{Comparison of alpha diversity difference index between groups}

\begin{tabular}{|llllllll|}
\hline index & Ace & Chao1 & $\begin{array}{l}\text { Goods } \\
\text { coverage }\end{array}$ & $\begin{array}{l}\text { Observed } \\
\text { species }\end{array}$ & $\begin{array}{l}\text { PD whole } \\
\text { tree }\end{array}$ & Shannon & Simpson \\
\hline $\begin{array}{l}P \\
\text { value }\end{array}$ & 0.00033 & 0.00035 & 0.000044 & 0.00098 & 0.00499 & 0.00021 & 0.00577 \\
\hline
\end{tabular}

\section{Beta diversity difference index}

The t-test was used to analyze whether the difference of species diversity among groups was significant. Based on Weighted Unifrac and Unweighted Unifrac distances, the results of difference analysis of beta diversity between two groups were represented by a boxplot (The upper two figures in Figure 5). In order to study the similarity of community composition, we performed Principal Co-ordinates Analysis (PCoA) based on weighted unifrac distance and unweighted unifrac distance (The lower two figures in Figure 5). The closer the sample distance is, the more similar the species composition.

\section{Functional prediction}

Based KEGG and COG datashops, we performed functional prediction of GM in each sample or group. The community structure component map shows the functional composition and relative abundance of each sample or group. TOP10 KEGG function items were selected to generate the relative abundance column accumulation diagram, as shown in figure 6. Membrane transport, carbohydrate metabolism, amino acid metabolism were first three pathway in function of GM. The COG functional prediction results were analyzed by Heatmap, as shown in figure 7. Moreover, STAMP results of COG was shown in figure 8.

Different colors represent different functional items, corresponding to the legend on the right; The horizontal axis represents different samples or groups, and the vertical axis represents the relative abundance of each functional item.

The horizontal axis represents different samples, and the vertical axis represents different functional items. The shade of color is related to the abundance of functional items. The darker the color, the higher the abundance.

The figure on the left shows the abundance ratio of different functions in the two samples or groups; the figure in the middle shows the difference ratio within the $95 \%$ confidence interval; the value on the far 
right is the $P$ value, $P$ value $<0.05$, indicating significant difference.

\section{Discussion}

In the last decades, using $16 \mathrm{~S}$ rDNA sequences, there have been an increasing amount of research into $\mathrm{GM}$, and it seems that most systemic diseases in the body are related to them [21].In our previous study, we found that there were differences in GM between irritable bowel syndrome patients and normal population in Nanchang, China [22]. In this study, we focused on the RSA patients and wanted to find the difference in GM between RSA patients and normal pregnant women in Nanchang, China. It is the first to analyze the differences in GM between RSA patients and normal pregnant women.

\section{The structure of gut microbiota changes during pregnancy}

The changes of GM in different stages of pregnancy have attracted much attention [23]. Studies from different regions have shown clear differences in the characteristics of GM between pregnant women and normal women. A US study reported that GM changed dramatically from first to third trimesters, and showed vast expansion of diversity between mothers, an overall increase in Proteobacteria and Actinobacteria [24]. A Sandi Arabia study indicated that bacterial diversity decreased in pregnant woman, whereas phylum Bacteroidetes declined significantly $(\mathrm{p}<0.05)$ in the first trimester, and a relatively high abundance of butyrate-producing bacteria (eg, Faecalibacterium spp. and Eubacterium spp.) in the gut of pregnant women [25]. A Japan research focused the differences in GM between early and late pregnancy, and they suggested that there were no obvious differences among four major phyla (Firmicutes, Bacteroidetes, Actinobacteria and Proteobacteria) between early and late pregnancy, although the proportion of the TM7 phylum decreased in late pregnancy compared with that in early pregnancy [26]. A study in China reported that at genus level, Akkermansia, Bacteroides, Subdoligranulum, Oscillospira, Ruminococcacea UCG-004), and Alistipes showed higher abundance during pregnancy [27].

\section{RSA patients have abnormal gut microbiota}

There has been no report on the correlation between RSA and GM, but there are many reports focused on the correlation between abortion and vaginal or oral bacteria. A China study was aimed at analyzing the changes in gut microorganism of patients with positive immune antibody-associated recurrent abortion using the 16s rRNA gene sequencing microbiome assay, and found that Bacteroides had the highest relative abundance in the positive group, Bacteroides, Erysipelotrichaceae_UCG-003, Faecalibacterium, and Prevotella_9 had high relative abundance in the negative group[28]. First trimester miscarriage associated with reduced prevalence of Lactobacillus spp.-dominated vaginal microbiota classified using hierarchical clustering analysis [29]. A study of vaginal microbiota showed that at the genus level, the relative abundance of Fam_Finegoldia and Lac_Roseburia significantly differed in the embryonic miscarriage group [30]. In the present study, although there was no significant difference in the abundance of dominant bacteria (Firmicutes, Bacteroidetes, Actinobacteria and Proteobacteria) between 
the two groups, but Actinobacteria and Proteobacteria tended to a higher in RSA patients at the phylum level. GM dysbiosis can cause insulin resistance (IR), which is closely linked to the occurrence of polycystic ovary syndrome (PCOS), and is associated with chronic inflammation, hormonal changes, follicular dysplasia, endometrial receptivity changes, and abortion or infertility [31].

\section{Roseburia and Agathobacter may have a protective effect against RSA}

In our study, one of the most striking result was a significant decrease in the abundance of genera Roseburia in the RSA group $(P<0.05)$. In addition, Agathobacterwas significantly increased in NR2 group, while Klebsiella $(P<0.05)$ were significantly increased, Roseburia $(P<0.05)$ and Prevotella. 9 $(P<0.05)$ were significantly decreased in RSA1 group. These results suggest that these bacteria play an important role in pregnancy, and the following studies can provide certain support for our research.

A Japan study in GM of infertile women showed that the abundance of the genera Roseburia and Phascolarctobacterium were decreased in patients with infertility [32]. A mice study indicated that the microbiota of conventional mice were significantly different at the end of pregnancy (day 18) as compared with pre-pregnancy $(\mathrm{p}<0.05)$, and the abundance of Roseburia faecis was significantly different at day 18 compared with pre-pregnancy [33].

Moreover, autism spectrum disorder (ASD) children with sleep disorder exhibited declines in the abundance of Agathobacter, decreased levels of 3-hydroxybutyric acid and melatonin [34]. Jin M et al found that Prevotella. 9 were significantly decreased in patients with positive immune antibodyassociated recurrent abortion [28]. The enrichment of Faecalibacterium, Agathobacter and Roseburia were related to geriatric depression [35].

\section{Gut mircobiota mechanism associated with RSA}

Functional prediction analysis indicated that GM may play their role through membrane transport, carbohydrate metabolism, amino acid metabolism and other mechanisms.

\section{Dietary composition:}

The composition of the GM can be influenced by dietary composition. In our study, Roseburia $(P<0.05)$ were significantly decreased in RSA group; furthermore, compared with RSA2 group, Roseburia $(P<0.05)$ and Prevotella. $9(P<0.05)$ were significantly decreased in RSA1 group (patients with BMI>23.9 in RSA). The human gut Firmicute Roseburia intestinalis is a primary degrader of dietary $\beta$-mannans, gut Roseburia spp. metabolize dietary components that stimulate their proliferation and metabolic activities. They are part of commensal bacteria producing short-chain fatty acids, especially butyrate, affecting 
colonic motility, immunity maintenance and anti-inflammatory properties [36, 37]. In early pregnancy, the relative abundances of Roseburia and Lachnospiraceae increased in GM of vegetarian compared with omnivorous diet, and sub-analysis of GM showed an alterations in fermentation end products from a mixed acid fermentation towards more acetate/butyrate [38].

\section{Immune maintenance and anti-inflammatory:}

Roseburia is one of the most important microorganisms in human intestinal tract, and its main metabolite is butyric acid. Of note, the GMs that we found to differ significantly at the genus level, such as Roseburia, Prevotella.9, Agathobacter, were butyrate producing bacteriums. A ulcerative colitis (UC) study suggested that butyrate-producing species like Roseburia hominis, involved in the development of UC [39]. Several animal studies reported that abundance of Roseburia intestinalis (R.I), decreased significantly in patients with inflammatory bowel disease (IBD) and exerted an anti-inflammatory function in dextran sulfate sodium (DSS)-induced colitis [40, 41, 42]. Another animal study on UC indicated that R.I flagellin plays an important role in the treatment of UC by inhibiting activation of the NLRP3 inflammasome and pyroptosis, [43]. In a murine model study suggested that Roseburia, a prominent gutassociated butyrate-producing bacterial genus, may provide a importion protection against atherosclerosis [44]. These studies suggest that R.I may be involved in the mechanism of immune maintenance and anti-inflammatory, which is related to butyrate.

\section{Butyrate-producing bacteria may prevent RSA by regulating BMI}

Previous evidence from animal studies suggests that butyrate-producing bacteria prevents high fat dietinduced obesity [45]. In the present study, the patients with BMI>23.9 in RSA have decreased Roseburia $(P<0.05)$ and Prevotella.9 $(P<0.05)$, both well-known butyrate-producing bacteria. Study suggested that chronic butyrate supplementation can prevent diet-induced obesity, hyperinsulinaemia, hypertriglyceridaemia and hepatic steatosis [46]. Abundances of butyrate-producing bacteria, such as R.I and Faecalibacterium prausnitzii, were lower in patients with type 2 diabetes (T2D). This result support that butyrate and other short-chain fatty acids are able to exert profound immunometabolic effects [47]. The abundance and butyrate-producing bacteria and butyrate production of overweight and obese women at 16 weeks gestation were significantly negatively correlated with blood pressure and plasminogen activator inhibitor-1 levels. These results suggest that increased butyrate production may help obese pregnant women maintain normal blood pressure. [48].

\section{Conclusion}

RSA patients have abnormal gut microbiota compared with normal pregnant women. Butyrate-producing bacteria, like Roseburia Prevotella.9, Agathobacter, maybe play an important role in pregnant women, and 
are associated with RSA. Our results provide the basis for in-depth studies of the composition of gut microbial communities in RSA.

\section{Abbreviations}

ASD: autism spectrum disorder; BMI: body mass index; COG: clusters of orthologous groups; GM: gut mircobiota; IBD: inflammatory bowel disease; IR: insulin resistance; KEGG: Kyoto encyclopedia of genes and genomes; NLRP3: NOD-like receptor protein 3; OTU: operational taxonomic units; PCA: principal component analysis; PCoA: principal co-ordinates analysis; PCOS: polycystic ovary syndrome; PCR: polymerase chain reaction; RSA: reccurent spontaneous abortion; T2D: type 2 diabetes; UC: ulcerative colitis; UPGMA: unweighted pair group method with arithmetic mean

\section{Declarations}

\section{Ethics approval and consent to participate}

All procedures complied with the principles of the Declaration of Helsinki, approved by our institutional review board and Ethics Committee of Jiangxi Maternal and Child Health Hospital, and documented in writing (No. 2020L005). Healthy controls were recruited among students and volunteers. All participants had signed informed consent.

\section{Consent for publication}

Not applicable.

\section{Availability of data and material}

All available data and material are in the manuscript and Supplementary Material.

\section{Competing interests}

All authors declare no conflict of interest of this work.

\section{Funding}


This study are supported by Jiangxi Provincial Key Laboratory of Female Reproduction integrated Traditional Chinese and Western Medicine.

\section{Authors' contributions}

YC and LH conceived the original idea of the study. LH analyzed data and wrote this paper. YC, QY, LZ, DL and HW collected cases and samples. All authors discussed the results and contributed to the final manuscript. All authors read and approved the final manuscript.

\section{Acknowledgments}

We thank Jiangxi University of Traditional Chinese Medicine (Nanchang, China) and APTBIO Technology (Shanghai, China) for its help.

\section{References}

1. Dethlefsen L, McFall-Ngai M, Relman DA. An ecological and evolutionary perspective on humanmicrobe mutualism and disease. Nature. 2007 Oct 18;449(7164):811-8. doi: 10.1038/nature06245. PMID: 17943117

2. Milani C, Duranti S, Bottacini F, et al. The First Microbial Colonizers of the Human Gut: Composition, Activities, and Health Implications of the Infant Gut Microbiota [J]. Microbiol Mol Biol Rev. 2017;81(4):e00036-17. doi: 10.1128/MMBR.00036-17. PMID: 29118049; PMCID: PMC5706746.

3. Vuillermin PJ, Macia L, Nanan R, et al. The maternal microbiome during pregnancy and allergic disease in the offspring. Semin Immunopathol [J]. 2017 Nov;39(6):669-675. doi: 10.1007/s00281017-0652-y. Epub 2017 Oct 16. PMID: 29038841; PMCID: PMC5711986.

4. Nyangahu DD, Lennard KS, Brown BP, et al. Disruption of maternal gut microbiota during gestation alters offspring microbiota and immunity [J]. Microbiome. 2018 Jul 7;6(1):124. doi: 10.1186/s40168018-0511-7. PMID: 29981583; PMCID: PMC6035804.

5. Nyangahu DD, Jaspan HB. Influence of maternal microbiota during pregnancy on infant immunity [J]. Clin Exp Immunol. 2019;198(1):47-56. doi: 10.1111/cei.13331. Epub 2019 Jun 21. PMID: $31121057 ;$ PMCID: PMC6718277.

6. Vandenplas Y, Carnielli VP, Ksiazyk J, et al. Factors affecting early-life intestinal microbiota development [J]. Nutrition. 2020 Oct;78:110812. doi: 10.1016/j.nut.2020.110812. Epub 2020 Mar 25. PMID: 32464473.

7. Penders J, Thijs C, Vink C, et al. Factors influencing the composition of the intestinal microbiota in early infancy. Pediatrics. 2006 Aug;118(2):511-21. doi: 10.1542/peds.2005-2824. PMID: 16882802. 
8. Corwin EJ, Hogue CJ, Pearce B, Hill CC, Read TD, Mulle J, Dunlop AL. Protocol for the Emory University African American Vaginal, Oral, and Gut Microbiome in Pregnancy Cohort Study. BMC Pregnancy Childbirth. 2017 Jun 1;17(1):161. doi: 10.1186/s12884-017-1357-x. Erratum in: BMC Pregnancy Childbirth. 2017 Nov 27;17 (1):395. PMID: 28571577; PMCID: PMC5455081.

9. Dunlop AL, Knight AK, Satten GA, Cutler AJ, Wright ML, Mitchell RM, Read TD, Mulle J, Hertzberg VS, Hill CC, Smith AK, Corwin EJ. Stability of the vaginal, oral, and gut microbiota across pregnancy among African American women: the effect of socioeconomic status and antibiotic exposure. PeerJ. 2019 Nov 21;7:e8004. doi: 10.7717/peerj.8004. PMID: 31772833; PMCID: PMC6875384.

10. Goltsman DSA, Sun CL, Proctor DM, et al. Metagenomic analysis with strain-level resolution reveals fine-scale variation in the human pregnancy microbiome. Genome Res. 2018 Oct;28(10):1467-1480. doi: 10.1101/gr.236000.118. Epub 2018 Sep 19. PMID: 30232199; PMCID: PMC6169887.

11. Benner M, Ferwerda G, Joosten I, et al. How uterine microbiota might be responsible for a receptive, fertile endometrium. Hum Reprod Update. 2018 Jul 1;24(4):393-415. doi: 10.1093/humupd/dmy012. PMID: 29668899.

12. Di Simone N, Santamaria Ortiz A, Specchia M, et al. Recent Insights on the Maternal Microbiota: Impact on Pregnancy Outcomes. Front Immunol. 2020 Oct 23;11:528202. doi: 10.3389/fimmu.2020.528202. PMID: 33193302; PMCID: PMC7645041.

13. Hu P, Chen X, Chu X, et al. Association of Gut Microbiota during Early Pregnancy with Risk of Incident Gestational Diabetes Mellitus. J Clin Endocrinol Metab. 2021 May 20:dgab346. doi: 10.1210/clinem/dgab346. Epub ahead of print. PMID: 34015117.

14. Chen X, Li P, Liu M, et al. Gut dysbiosis induces the development of pre-eclampsia through bacterial translocation. Gut. 2020 Mar;69(3):513-522. doi: 10.1136/gutjnl-2019-319101. Epub 2020 Jan 3. PMID: 31900289

15. Practice Committee of the American Society for Reproductive Medicine. Evaluation and treatment of recurrent pregnancy loss: a committee opinion. Fertil Steril. 2012;98:1103-11. doi: 10.1016/j.fertnstert.2012.06.048. Epub 2012 Jul 24. PMID: 22835448.

16. Royal College of Obstetricians and Gynaecologists. The investigation and treatment of couples with recurrent frst-trimester and second-trimester miscarriage (Green-top Guideline No. 17). 2011 [EB/OL]. https://www.rcog.org.uk/globalassets/documents/guidelines/gtg_17.pdf.

17. Cui Y, He L, Yang CY, et al. iTRAQ and PRM-based quantitative proteomics in early recurrent spontaneous abortion: biomarkers discovery. Clin Proteomics. 2019 Oct 18;16:36. doi: 10.1186/s12014-019-9256-y. PMID: 31636515; PMCID: PMC6798364.

18. Fan $T$, Zhong XM, Wei $X C$, et al. The alteration and potential relationship of vaginal microbiota and chemokines for unexplained recurrent spontaneous abortion. Medicine (Baltimore). 2020 Dec 18;99(51):e23558. doi: 10.1097/MD.0000000000023558. PMID: 33371084; PMCID: PMC7748213.

19. Lundberg DS, Yourstone S, Mieczkowski P, et al. Practical innovations for high-throughput amplicon sequencing. Nat Methods. 2013 Oct;10(10):999-1002. doi: 10.1038/nmeth.2634. Epub 2013 Sep 1. PMID: 23995388. 
20. Avershina E, Frisli T, Rudi K. De novo semi-alignment of 16S rRNA gene sequences for deep phylogenetic characterization of next generation sequencing data. Microbes Environ. 2013;28(2):211-6. doi: 10.1264/jsme2.me12157. Epub 2013 Apr 20. PMID: 23603801; PMCID: PMC4070667.

21. Lynch SV, Pedersen O. The Human Intestinal Microbiome in Health and Disease. N Engl J Med. 2016 Dec 15;375(24):2369-2379. doi: 10.1056/NEJMra1600266. PMID: 27974040.

22. Mei L, Zhou J, Su Y, et al. Gut microbiota composition and functional prediction in diarrheapredominant irritable bowel syndrome. BMC Gastroenterol. 2021 Mar 5;21(1):105. doi: 10.1186/s12876-021-01693-w. PMID: 33663411; PMCID: PMC7934555.

23. Gosalbes MJ, Compte J, Moriano-Gutierrez S, et al. Metabolic adaptation in the human gut microbiota during pregnancy and the first year of life. EBioMedicine. 2019 Jan;39:497-509. doi: 10.1016/j.ebiom.2018.10.071. Epub 2018 Nov 8. PMID: 30415891; PMCID: PMC6354444.

24. Koren $\mathrm{O}$, Goodrich JK, Cullender TC, et al. Host remodeling of the gut microbiome and metabolic changes during pregnancy. Cell. 2012 Aug 3;150(3):470-80. doi: 10.1016/j.cell.2012.07.008. PMID: 22863002; PMCID: PMC3505857

25. Khan I, Yasir M, Farman M, et al. Evaluation of gut bacterial community composition and antimicrobial resistome in pregnant and non-pregnant women from Saudi population. Infect Drug Resist. 2019 Jun 21;12:1749-1761. doi: 10.2147/IDR.S200213. PMID: 31417292; PMCID: PMC6593780.

26. Sakurai K, Kato T, Tanabe H, et al. Association between gut microbiota composition and glycoalbumin level during pregnancy in Japanese women: Pilot study from Chiba Study of Mother and Child Health. J Diabetes Investig. 2020 May;11(3):699-706. doi: 10.1111/jdi.13177. Epub 2019 Dec 28. Erratum in: J Diabetes Investig. 2021 Mar;12(3):462. PMID: 31721478; PMCID: PMC7232291.

27. Qin S, Liu Y, Wang S, et al. Distribution characteristics of intestinal microbiota during pregnancy and postpartum in healthy women. J Matern Fetal Neonatal Med. 2021 Feb 4:1-8. doi:

10.1080/14767058.2020.1812571. Epub ahead of print. PMID: 33541190.

28. Jin M, Li D, Ji R, et al. Changes in Gut Microorganism in Patients with Positive Immune AntibodyAssociated Recurrent Abortion. Biomed Res Int. 2020 Sep 18;2020:4673250. doi: 10.1155/2020/4673250. PMID: 33015167; PMCID: PMC7520699.

29. Chang DH, Shin J, Rhee MS, et al. Vaginal Microbiota Profiles of Native Korean Women and Associations with High-Risk Pregnancy. J Microbiol Biotechnol. 2020 Feb 28;30(2):248-258. doi: 10.4014/jmb.1908.08016. PMID: 31838792.

30. Xu L, Huang L, Lian C, et al. Vaginal Microbiota Diversity of Patients with Embryonic Miscarriage by Using 16S rDNA High-Throughput Sequencing. Int J Genomics. 2020 Nov 20;2020:1764959. doi: 10.1155/2020/1764959. PMID: 33299847; PMCID: PMC7704210.

31. He FF, Li YM. Role of gut microbiota in the development of insulin resistance and the mechanism underlying polycystic ovary syndrome: a review. J Ovarian Res. 2020 Jun 17;13(1):73. doi: 
10.1186/s13048-020-00670-3. PMID: 32552864; PMCID: PMC7301991.

32. Komiya S, Naito $\mathrm{Y}, \mathrm{Okada} \mathrm{H}$, et al. Characterizing the gut microbiota in females with infertility and preliminary results of a water-soluble dietary fiber intervention study. J Clin Biochem Nutr. 2020 Jul;67(1):105-111. doi: 10.3164/jcbn.20-53. Epub 2020 Jun 5. PMID: 32801476; PMCID: PMC7417798.

33. Faas MM, Liu Y, Borghuis T, et al. Microbiota Induced Changes in the Immune Response in Pregnant Mice. Front Immunol. 2020 Jan 9;10:2976. doi: 10.3389/fimmu.2019.02976. PMID: 31998293; PMCID: PMC6962187.

34. Hua X, Zhu J, Yang T, et al. The Gut Microbiota and Associated Metabolites Are Altered in Sleep Disorder of Children With Autism Spectrum Disorders. Front Psychiatry. 2020 Sep 2;11:855. doi: 10.3389/fpsyt.2020.00855. PMID: 32982808; PMCID: PMC7493623.

35. Lee SM, Dong TS, Krause-Sorio B, et al. The intestinal microbiota as a predictor for antidepressant treatment outcome in geriatric depression: a prospective pilot study. Int Psychogeriatr. 2021 Mar 24:1-13. doi: 10.1017/S1041610221000120. Epub ahead of print. PMID: 33757609.

36. Tamanai-Shacoori Z, Smida I, et al. Roseburia spp.: a marker of health? Future Microbiol. 2017 Feb;12:157-170. doi: 10.2217/fmb-2016-0130. PMID: 28139139.

37. La Rosa SL, Leth ML, Michalak L, et al. The human gut Firmicute Roseburia intestinalis is a primary degrader of dietary $\beta$-mannans. Nat Commun. 2019 Feb 22;10(1):905. doi: 10.1038/s41467-01908812-y. PMID: 30796211; PMCID: PMC6385246.

38. Barrett HL, Gomez-Arango LF, Wilkinson SA, et al. A Vegetarian Diet Is a Major Determinant of Gut Microbiota Composition in Early Pregnancy. Nutrients. 2018 Jul 12;10(7):890. doi: 10.3390/nu10070890. PMID: 30002323; PMCID: PMC6073691.

39. Machiels K, Joossens M, Sabino J, et al. A decrease of the butyrate-producing species Roseburia hominis and Faecalibacterium prausnitzii defines dysbiosis in patients with ulcerative colitis. Gut. 2014 Aug;63(8):1275-83. doi: 10.1136/gutjnl-2013-304833. Epub 2013 Sep 10. PMID: 24021287.

40. Shen Z, Zhu C, Quan Y, et al. Insights into Roseburia intestinalis which alleviates experimental colitis pathology by inducing anti-inflammatory responses. J Gastroenterol Hepatol, 2018; 33: 1751-1760. doi: 10.1111/jgh.14144. Epub 2018 May 7. PMID: 29532517.

41. Tan B, Luo W, Shen Z, et al. Roseburia intestinalis inhibits oncostatin $M$ and maintains tight junction integrity in a murine model of acute experimental colitis. Scand J Gastroenterol. 2019 Apr;54(4):432440. doi: 10.1080/00365521.2019.1595708. Epub 2019 Apr 4. PMID: 30946611.

42. Zhu C, Song K, Shen Z, et al. Roseburia intestinalis inhibits interleukin 17 excretion and promotes regulatory T cells differentiation in colitis. Mol Med Rep, 2018; 17: 7567-7574. doi: 10.3892/mmr.2018.8833. Epub 2018 Mar 29. PMID: 29620246; PMCID: PMC5983956.

43. Wu X, Pan S, Luo W, et al. Roseburia intestinalis-derived flagellin ameliorates colitis by targeting miR-223-3p-mediated activation of NLRP3 inflammasome and pyroptosis. Mol Med Rep. 2020 Oct;22(4):2695-2704. doi: 10.3892/mmr.2020.11351. Epub 2020 Jul 23. PMID: 32700754; PMCID: PMC7453595. 
44. Kasahara K, Krautkramer KA, Org E, et al. Interactions between Roseburia intestinalis and diet modulate atherogenesis in a murine model. Nat Microbiol. 2018 Dec;3(12):1461-1471. doi: 10.1038/s41564-018-0272-x. Epub 2018 Nov 5. PMID: 30397344; PMCID: PMC6280189.

45. Bai L, Gao M, Cheng $X$, et al. Engineered butyrate-producing bacteria prevents high fat diet-induced obesity in mice. Microb Cell Fact. 2020 Apr 25;19(1):94. doi: 10.1186/s12934-020-01350-z. PMID: 32334588; PMCID: PMC7183672.

46. Li Z, Yi CX, Katiraei S, et al. Butyrate reduces appetite and activates brown adipose tissue via the gutbrain neural circuit. Gut. 2018 Jul;67(7):1269-1279. doi: 10.1136/gutjnl-2017-314050. Epub 2017 Nov 3. PMID: 29101261.

47. Tilg H, Moschen AR. Microbiota and diabetes: an evolving relationship. Gut. 2014 Sep;63(9):1513-21. doi: 10.1136/gutjnl-2014-306928. Epub 2014 May 15. PMID: 24833634.

48. Gomez-Arango LF, Barrett HL, Mclntyre HD, et al. Increased Systolic and Diastolic Blood Pressure Is Associated With Altered Gut Microbiota Composition and Butyrate Production in Early Pregnancy. Hypertension. 2016 Oct;68(4):974-81. doi: 10.1161/HYPERTENSIONAHA.116.07910. Epub 2016 Aug 15. PMID: 27528065.

\section{Figures}

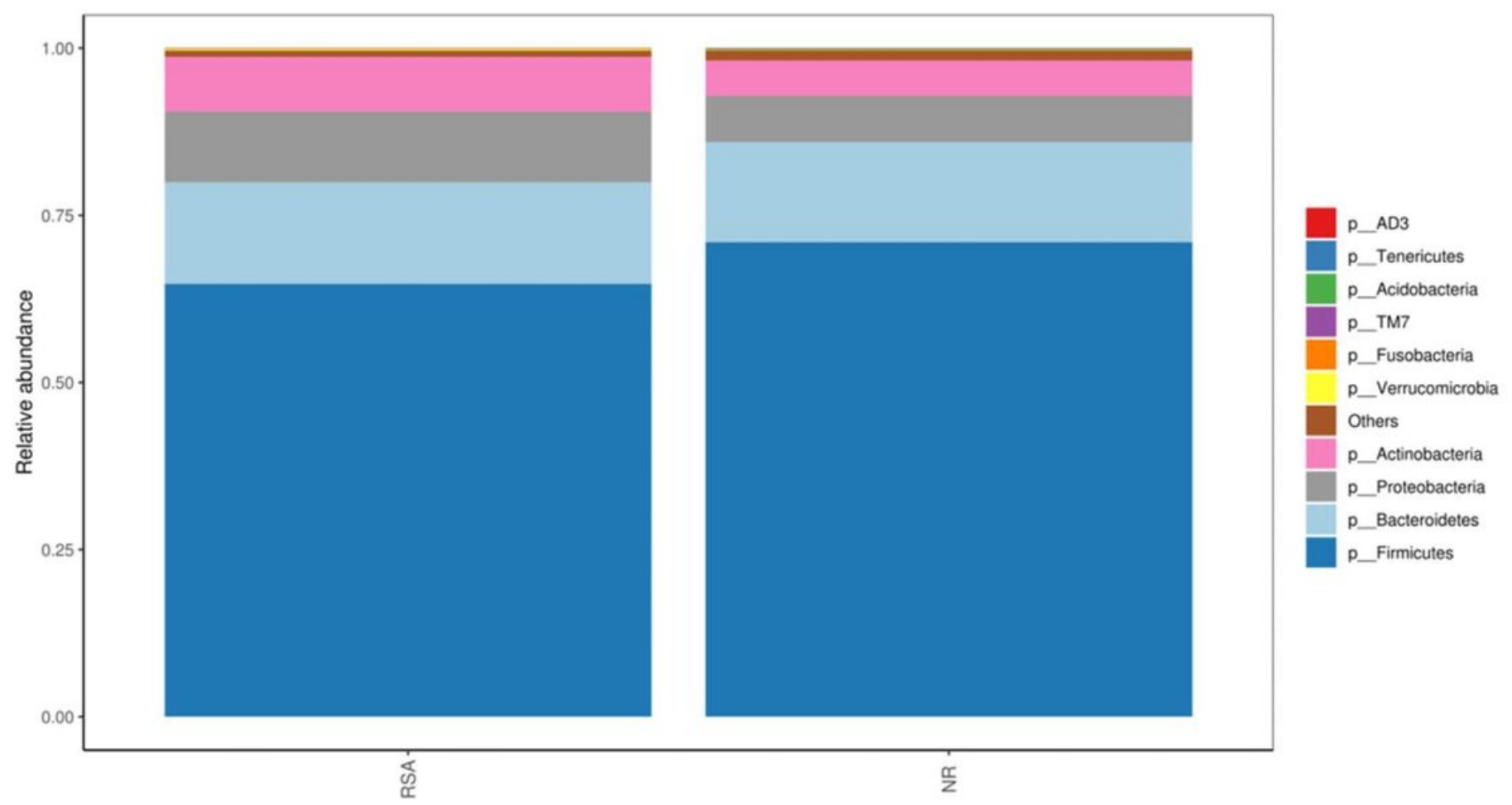

\section{Figure 1}

Top10 histogram of relative abundance of species (phylum level) 


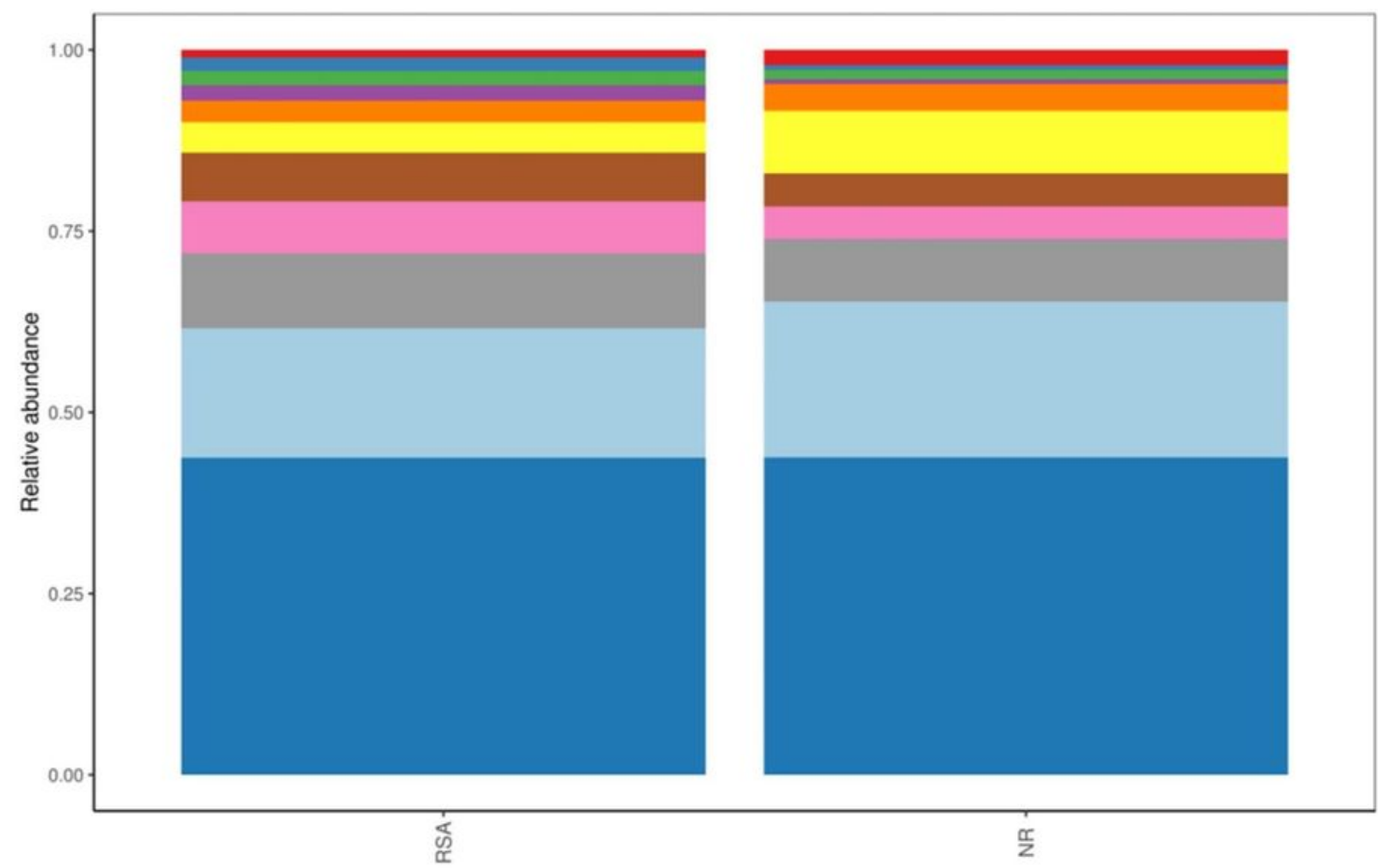

g_Dialister

g_ SMB53

g_Coprococcus

g_Ruminococcus.

g. Prevotella

g Roseburia

g_Blautia

g Bifidobacterium

g. Bacteroides

g_ Faecalibacterium

Others

\section{Figure 2}

Top10 histogram of relative abundance of species (Genus level)

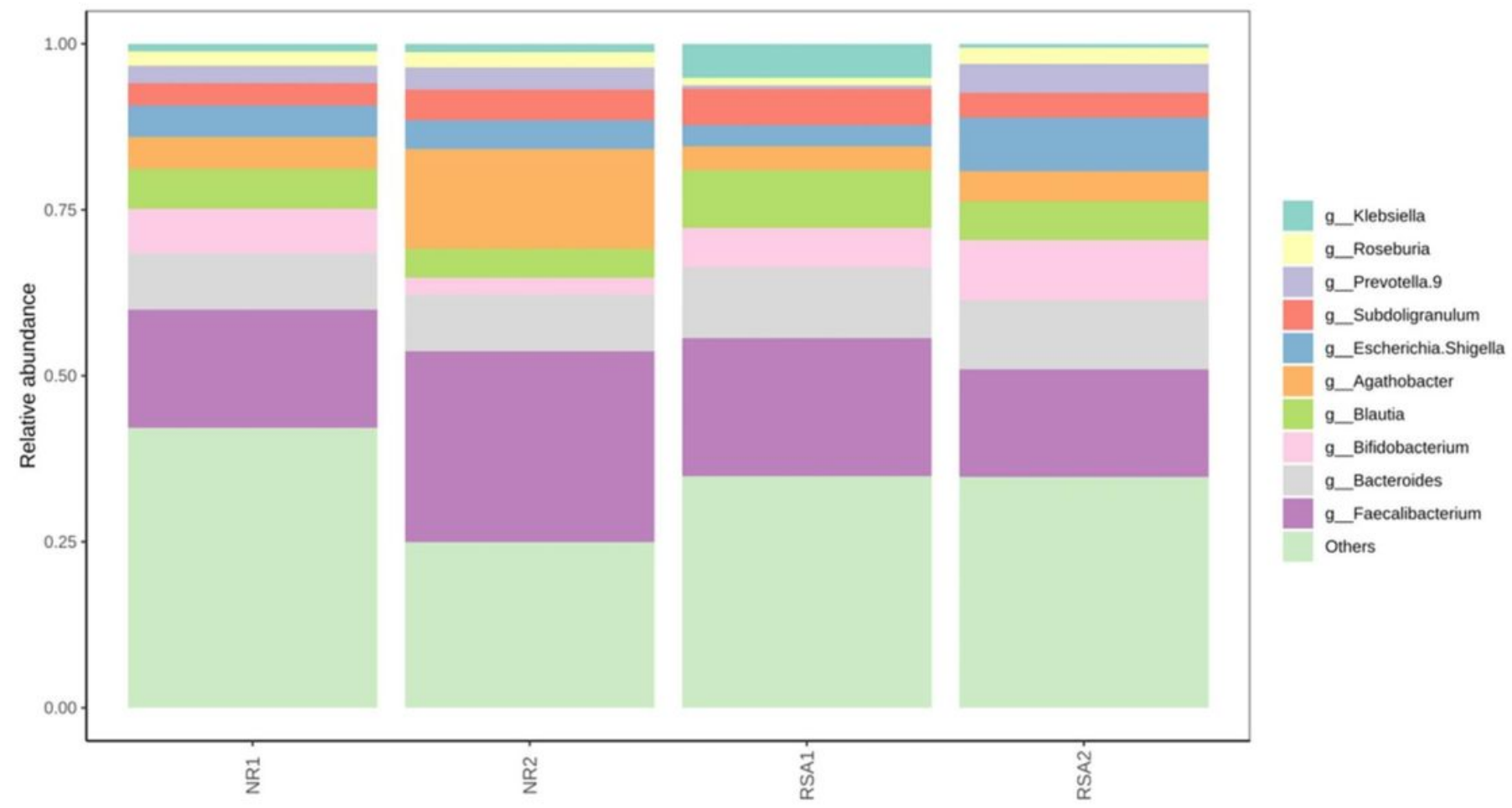

Figure 3

Top10 histogram of relative abundance of species in four groups (Genus level) 

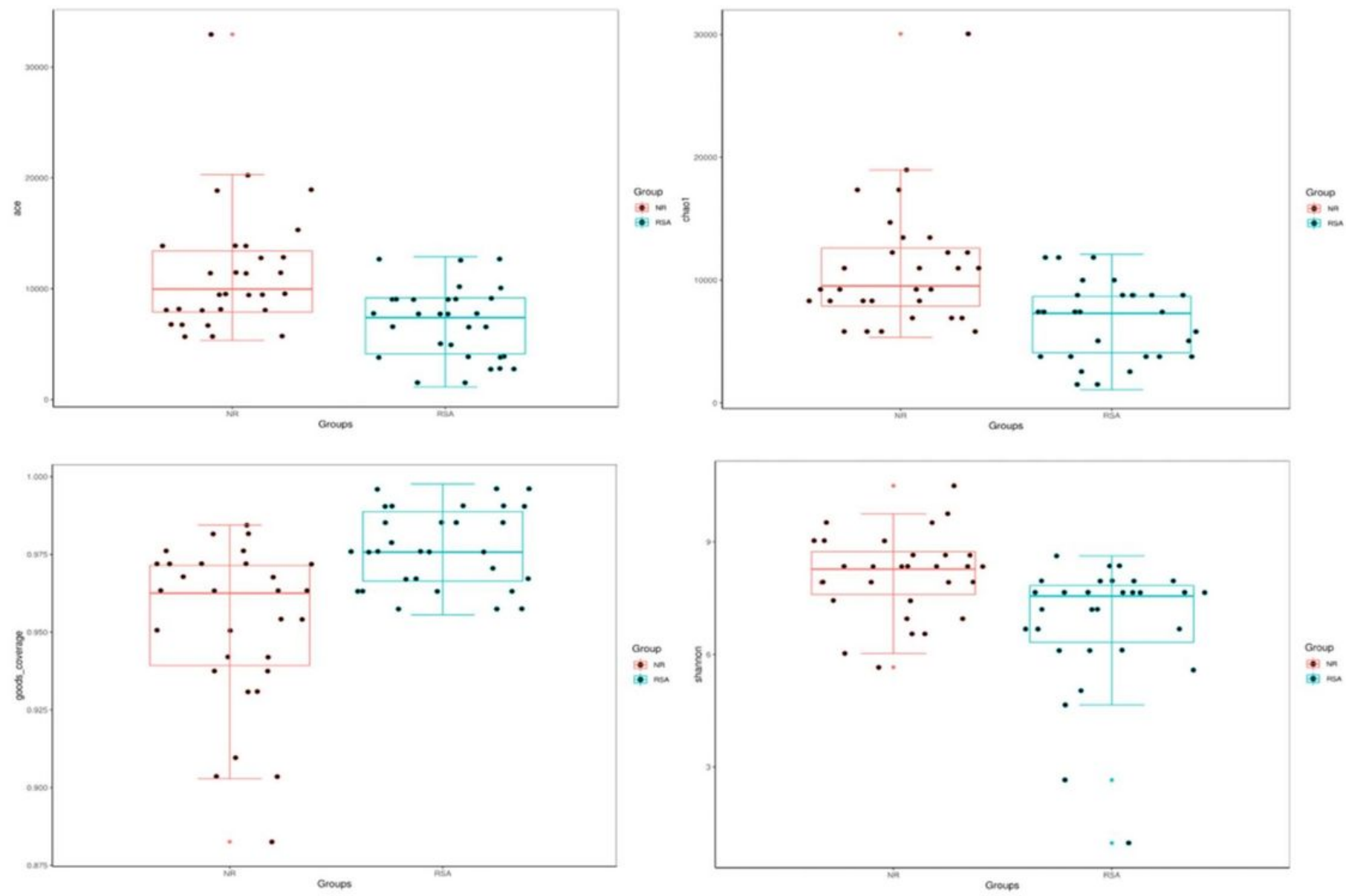

Figure 4

Boxplot of Alpha diversity index between RSA and NR 

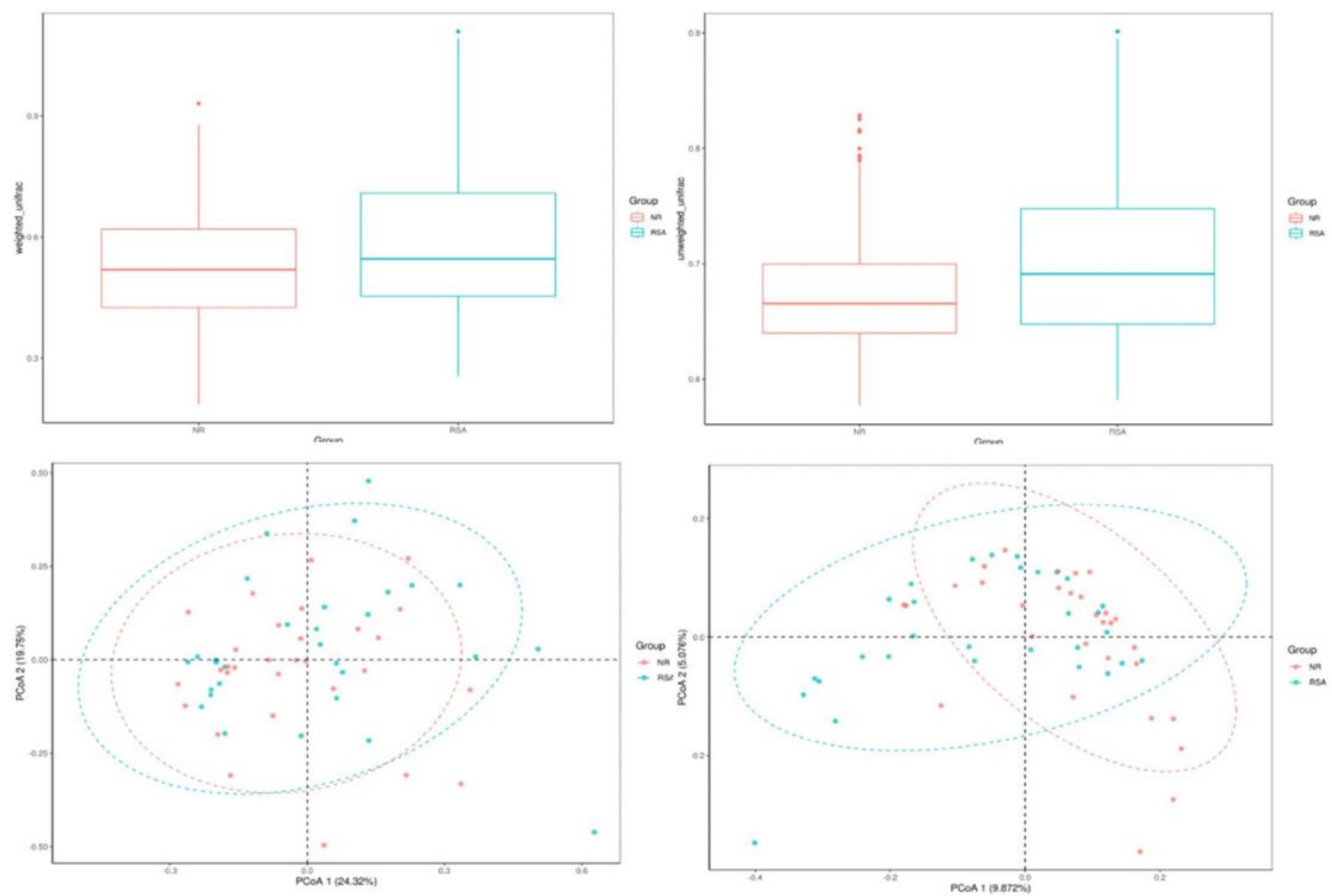

Figure 5

Characteristics of Beta diversity index between RSA and NR 


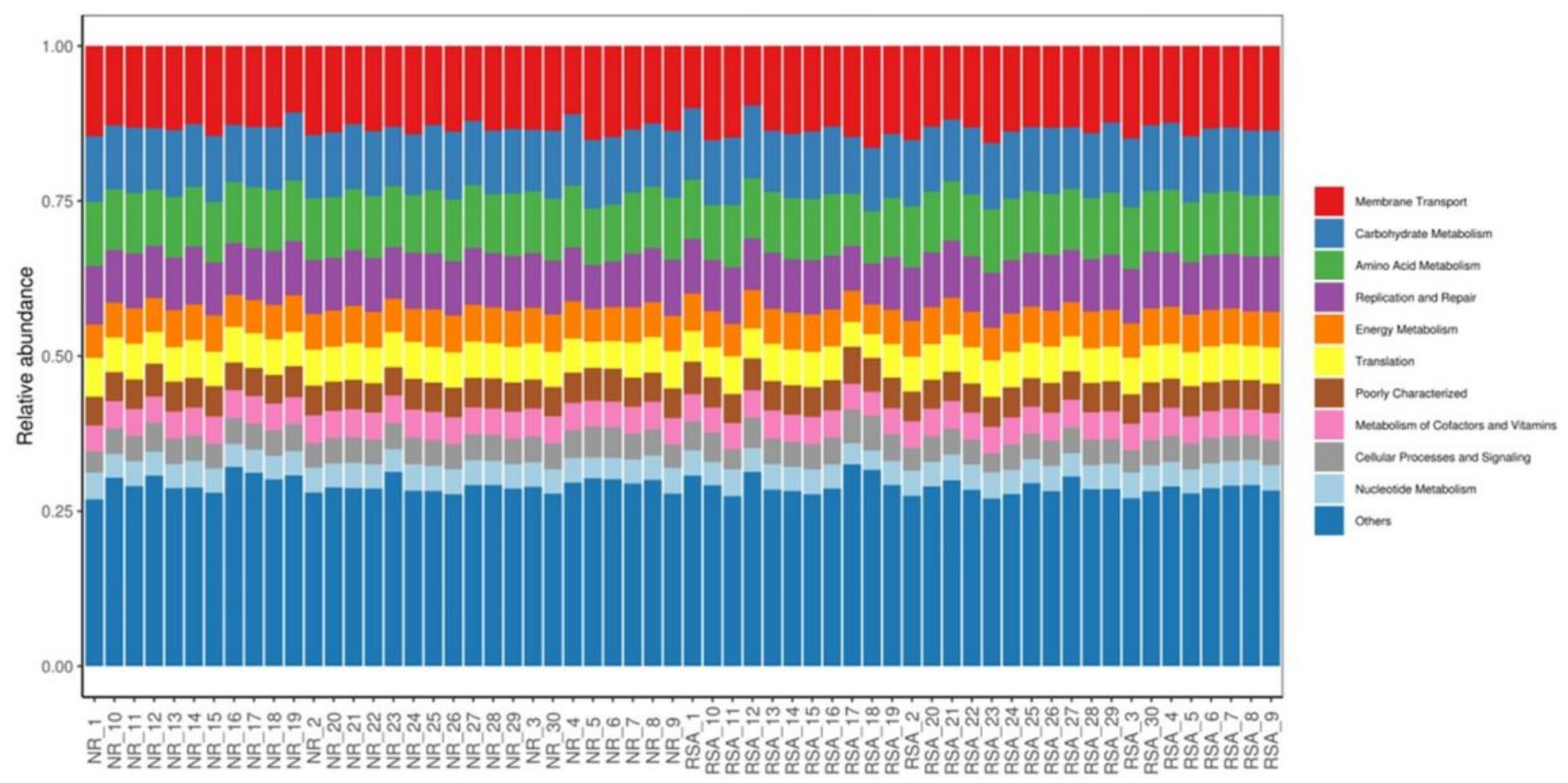

Figure 6

Barchart of relative abundance of KEGG functional items 


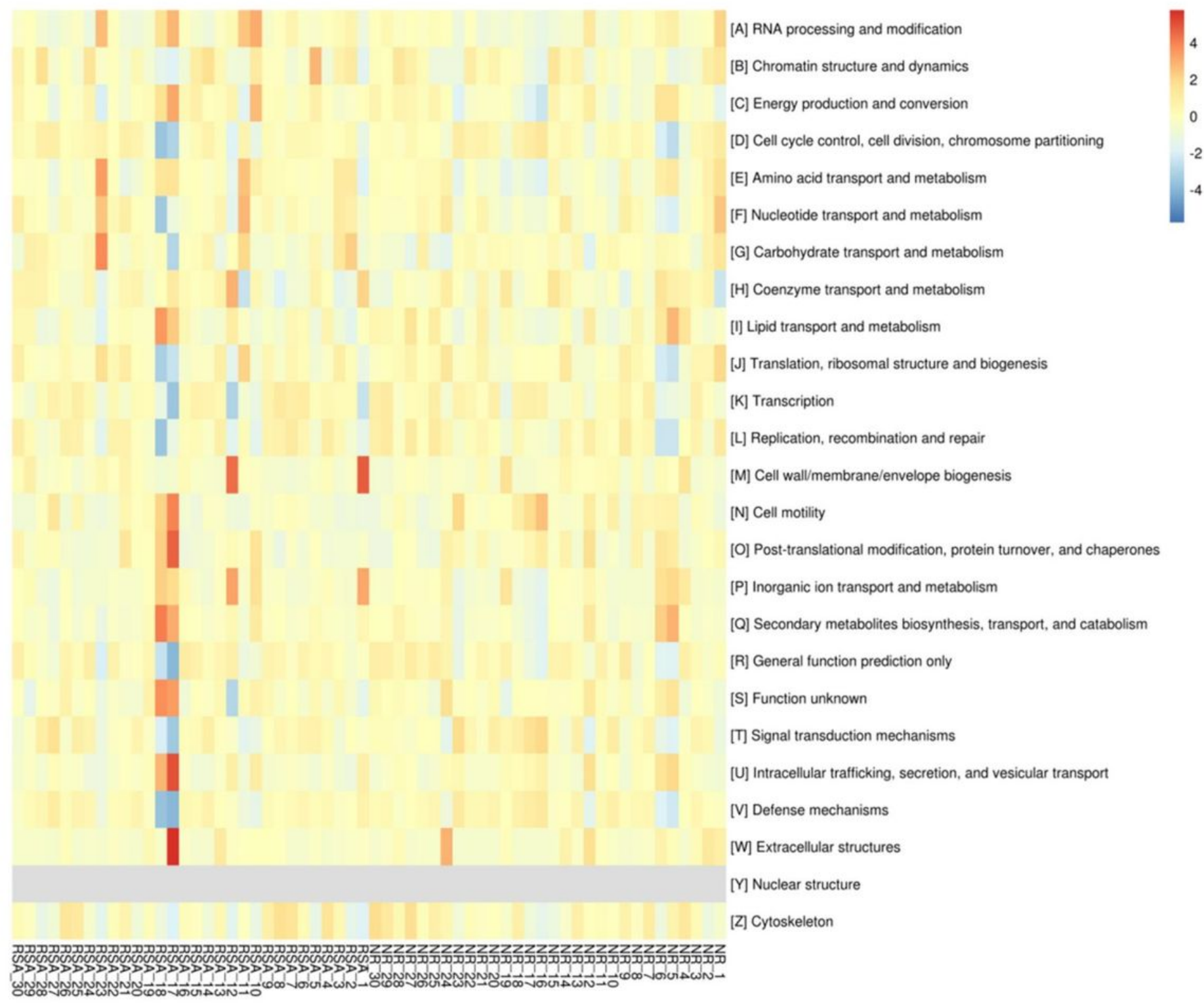

Figure 7

Functional annotation clustering heatmap of COG 

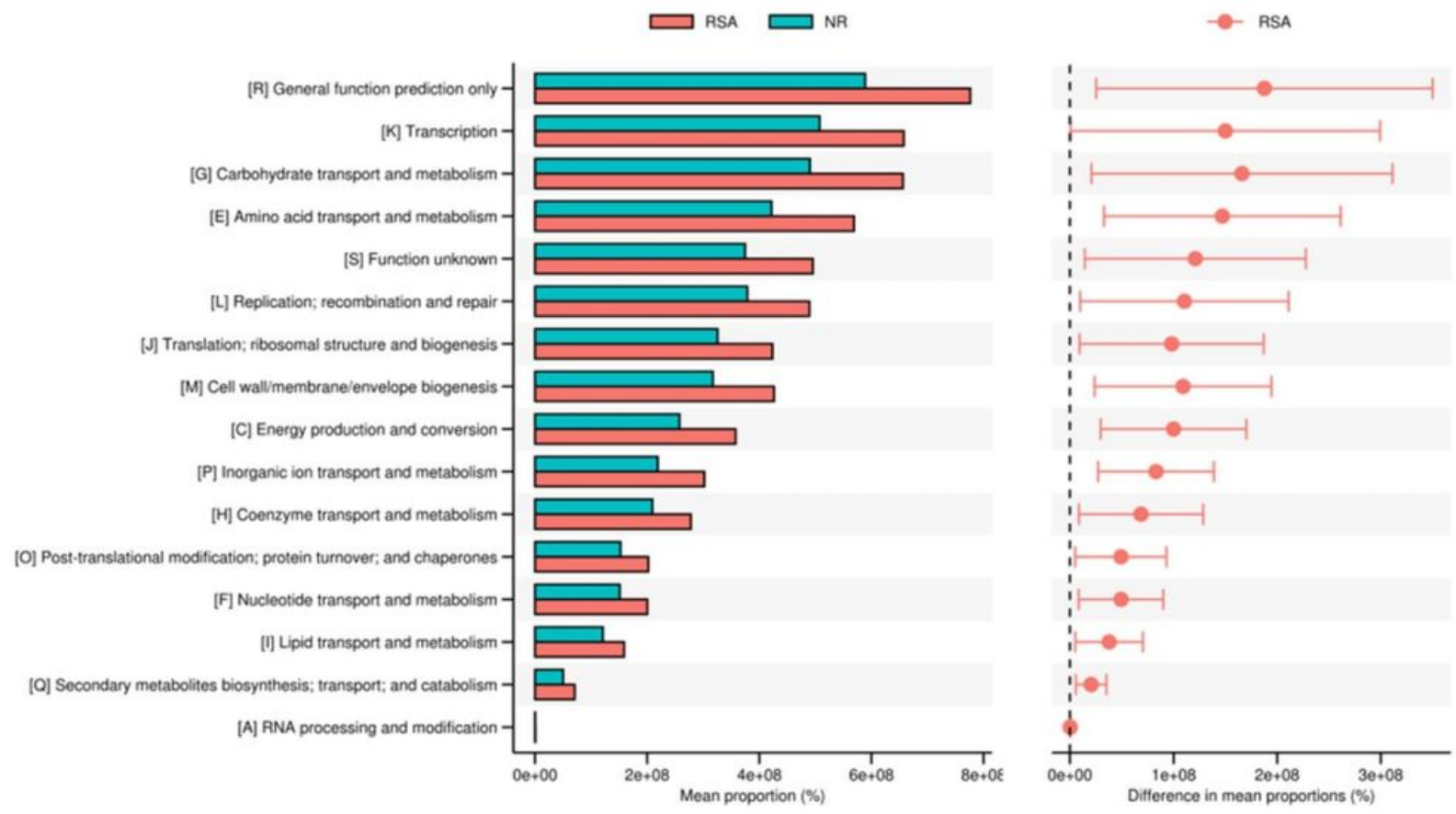

0.0244

0.0490

0.0259

0.0126

0.0269

0.0322

0.0311

0.0132 옹

0.0063 a

0.0044

0.0257

0.0292

0.0190

0.0239

0.0076

0.0327

\section{Figure 8}

STAMP results of COG

\section{Supplementary Files}

This is a list of supplementary files associated with this preprint. Click to download.

- 1characteristicoftwogroups.xls

- 2comparisonoftwogroupsatphylum.xlsx

- 3comparisonoftwogroupsatgenus.xlsx

- 4comparisonoffourgroupsatphylum.xlsx

- 5comparisonoffourgroupsatgenus.xIsx

- 6alphadiversityindex.xIsx

- 7keggpredictedL2.xIsx

- 8cogpredictedL2.xlsx 